\title{
Non-Hodgkins Lymphoma Presenting as a Skull Metastases: Case Report
}

\author{
Yam Bahadur Roka ${ }^{1}$, Narayani Roka ${ }^{2}$, Mohan Karki ${ }^{1}$ \\ ${ }^{1}$ Department of Neurosurgery, Neuro Cardio and Multispecialty Hospital, Biratnagar, Nepal \\ ${ }^{2}$ Department of Ophthalmology, Neuro Cardio and Multispecialty Hospital, Biratnagar, Nepal
}

Correspondence:

Dr. Yam B Roka M.S, M.Ch (Neurosurgery), FNNI (Skull Base Surgery), IFAANS

Chief of Neurosurgery and Medical Director

Neuro Cardio and Multispecialty Hospital

Jahada Road, Biratnagar-13, Nepal

Email: dryamroka@yahoo.com

Phone: +9779841256560

\begin{abstract}
The incidence of cerebral metastases has increased over the last few decades mainly due to the successful treatment of extra neural cancers and the prolonged survival of patients. The common causes of metastases are lung, kidney, breast and thyroid cancers. We present an interesting case of cerebral metastases that was managed as cerebral abscess, then as lung metastases before finally arriving to the correct diagnosis of Non-Hodgkin's Lymphoma.
\end{abstract}

Key Words: Cancer, Cerebral abscess, Cerebral metastases, Non-Hodgkin's Lymphoma

I $\mathrm{n}$ cases with systemic cancer the incidence of cerebral metastases has increased over the last few decades with an incidence of $20-25 \%{ }^{1}$ The common causes of metastases are lung, kidney, breast and thyroid cancers. ${ }^{2}$ We present an interesting case of cerebral metastases that was managed initially as cerebral abscess, followed by lung metastases before finally being diagnosed as a case of Non-Hodgkin's lymphoma (NHL)

\section{Case Description:}

A 63-year-old female presented to the hospital with history of a progressively enlarging mass over the right frontal area for the last three months. There was history of minor trauma to the site along with few spells of fever, nausea and abdominal pain. She had been managed elsewhere as a case of localized abscess with antibiotics, analgesics and antiemetic's with no relief in symptoms. There was no history of any other illness in the past except being a heavy smoker.
On examination she was alert, conscious with Glasgow Coma Score of 15. There was a $5 X 5 \mathrm{~cm}$ mass over the right frontal area which was firm; non-tender, fixed, non-pulsatile and non-reducible (Figure 1A). There were no other cranial nerves or systemic deficits. The routine bloods showed raised total leucocyte count and serology for hepatitis B \& $\mathrm{C}$ and human immunodeficiency virus was negative. A possible case of osteomyelitis was kept in diagnosis. Chest Skiagram showed a right paramediastinal mass which was assumed to be carcinoma lung in view of the history of heavy smoking (Figure 1B). Computed tomogram (CT) of the head showed heterogeneously enhancing right frontal dumbbell mass which was extending intracranially to the epidural space (Figure 1C). There was no other intracerebral lesion or infiltration seen. A possibility of cerebral metastases secondary to lung cancer was now kept as the differential diagnosis. 


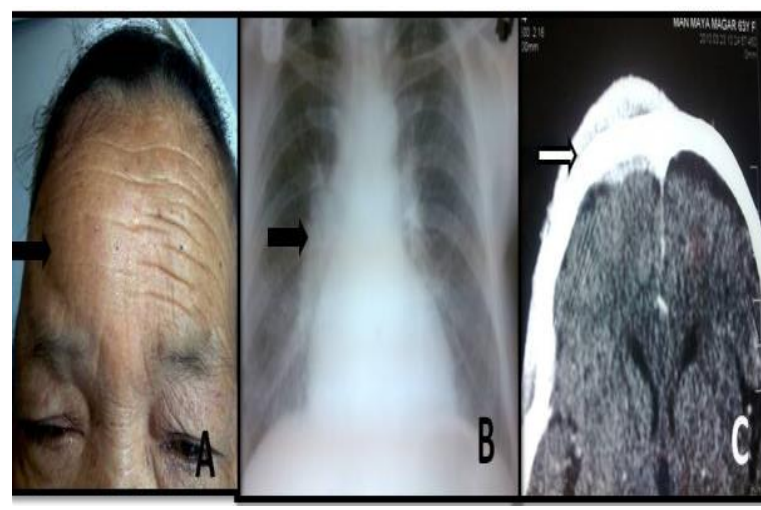

Figure 1: Clinical picture showing the right frontal mass (A), the right mediastinal mass on chest skiagram (B) and CT head showing a right frontal mass with extension to epidural space (C).

She was being planned for fine needle aspiration (FNAC) of the frontal mass and also CT guided aspiration of the thoracic mass when she complained of diarrhea with severe pain abdomen. Per abdomen examination revealed multiple firm intraabdominal masses, non-tender, partially mobile with no hepatosplenomegaly. Ultrasound revealed multiple matted para-aortic masses and nodes along the iliac and femoral vessels (Figure 2).

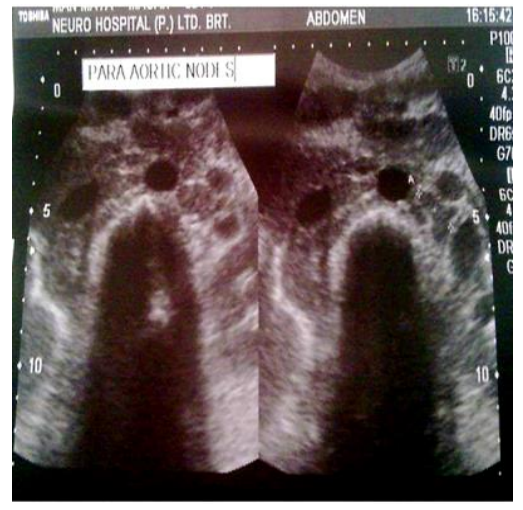

Figure 2: Ultrasound showing large bilateral multiple matted paraaortic lymph nodes.

FNAC was done of the most superficial lump in the abdomen and the frontal mass, which revealed it to be Non-Hodgkin's lymphoma (NHL). She was advised surgical excision of the frontal mass with cranioplasty but she refused. Grade IV NHL was the final diagnosis and she was referred to the oncologist for further $\mathrm{CD}$ marker studies and management.

egneuro Volume 03, Issue 01, 2021

\section{Discussion}

NHL occurs with incidence of $3-4 \%$ and involvement of the central nervous system occur in $1-2 \%$ of cases only. ${ }^{4}$ The incidence of cerebral metastases has increased over the last few decades mainly due to the successful treatment of extra neural cancers and the prolonged survival of patients. NHL can affect the nervous system in several ways that may be direct involvement of the leptomeninges, by metastases or secondary to paraneoplastic syndrome. ${ }^{4-6}$ The common symptoms of nervous system involvement are neurological deficits, headache, localized mass or seizures which can be in addition to general symptoms of fatigue, fever, loss of weight, pain abdomen or respiratory difficulties. These vague symptoms and signs are often misinterpreted as milder disease and thus present a difficult diagnostic and management problems to the attending physician. In addition, patients may develop infectious complications of the nervous system from immunosuppression and use of antineoplastic therapies related to the disease or its treatment. ${ }^{7}$ CT or MRI can clinch the diagnosis which show contrast enhancing lesions which are isodense or isointense in plain scans and improve detection with short tau inversion recovery imaging. ${ }^{8}$

This case is interesting in the view that there were three differentials, abscess, lung carcinoma and secondary from the lung carcinoma which was made before the final diagnosis was confirmed. Apart from mild fever and diarrhea, there were no other systemic manifestations. The lessons learnt from this case is that, lumps with even with a history of trauma may not abscess, even in smoker's lung mass may be secondary to some other pathology and carcinoma lung although one of the commonest causes may not be the cause for all cases of cerebral metastases. Finally, the abdomen remains one of the sites to be ruled for primary in all cases of cerebral metastases. This is important in the developing countries where cases like these are misdiagnosed and managed at periphery for prolonged periods leading to progression of the disease during final diagnosis.

The best treatment of NHL once the diagnosis is confirmed is the use of systemic chemotherapy 
along with intrathecal or radiotherapy to control the disease, palliation and prolong survival. In specialized centers there is the option of high-dose systemic chemotherapy with hematopoietic stem cell transplantation in those cases that do not have active central nervous system disease. ${ }^{9}$

\section{Conclusion:}

All cases with swelling of the scalp are not abscess/osteomyelitis/epidermoid/ dermoid and thus thorough search must be made systemically to rule out other primary source. ${ }^{10}$ The abdomen and chest remains the Pandora's box and must be examined in all cases with suspected central nervous system lesions that do not improve with first line medical treatment.

\section{References:}

1. Suh JH, Chao ST, Vogelbaum MA. Management of brain metastases. Curr Neurol Neurosci Rep. 2009; 9(3):223230. [Pubmed]

2. Roka YB, Pandey SR, Bhattarai NR. Solitary Skull Metastases from Breast Carcinoma. Nepal Journal of Neuroscience. 2018 May 28; 15(1):38-9. [Article]

3. Grier J, Batchelor T. Metastatic neurologic complications of non-Hodgkin's lymphoma. Curr Oncol Rep. 2005; 7(1):55-60. [Pubmed]

4. Jaiswal M, Gandhi A, Purohit D, Singhvi S, Mittal RS. Primary non-Hodgkin's lymphoma of the skull with extra and intracranial extension presenting with bulky scalp mass lesion. Asian journal of neurosurgery. 2016 Oct;11(4):444. [Pubmed]

5. Recht LD. Neurologic complications of systemic lymphoma. Neurol Cli. 1991; 9(4):1001-15. [Pubmed]

6. Giglio P, Gilbert MR. Neurologic complications of nonHodgkin's lymphoma. Curr Oncol Rep. 2005; 7(1):61-5. [Pubmed]

7. Glass J. Neurologic complications of lymphoma and leukemia. Semin Oncol. 2006; 33(3):342-7. [Pubmed]

8. Roug IK, McCartney LB. Metastatic non-Hodgkin lymphoma presenting as low back pain and radiculopathy: a case report. Journal of chiropractic medicine. 2012 Sep 1; 11(3):202-6. [Pubmed]

9. Bierman P, Giglio P. Diagnosis and treatment of central nervous system involvement in non-Hodgkin's lymphoma. Hematol Oncol Clin North Am. 2005; 19(4):597-609. [Pubmed]

10. Roka YB, Bajracharya A, Shrestha K, Khaniya S. Parietal Epidural Dermoid Mimicking a Scalp Abscess in an Infant: Case Report. Journal of Nepal Paediatric Society. 2009 Jul 16; 29(2):98-100. [Article] 